\title{
Digital Image Processing Assessment in Multi Slice CT Angiogram using Liner, Non-Liner and Both Classifiers
}

\author{
Sarvadeva BhatlaMurali Krishna*, Dr. M Chandra Shekar** \\ *(PhD Scholar in ECE, at Rayalaseema University with Regd No. PP-ECE-0044, AP, India) \\ **(Senior Manager Bharath Dynamics Limited, Hyderabad, Telangana, India)
}

\begin{abstract}
Nowadays with heart diseases most of peoples are dying lock process to find problem agile fashion in remote areas. In this research help to peoples who are staying remote also to find problem in heart on which location and how much problem to near and finally gives best analysis methodology for automated analysis for MultiSlice CT Angiogram images. Multi slice CT scanner is used to identify heart disease. Multi-detector CT is considered convenient and reliable non-invasive imaging modality for assessment of human angiogram 3D images. Automatic hart segmentation from Computed Tomography (CT) is highly demanded. Accurate hart segmentation is a crucial for computer-aided heart disease diagnosis and treatment planning. After segmentation and future extraction then identify whether the patient angiogram waveform has disease or not. For that, Support Vector Machine method is used to confirm the presence of disease. Neural Networks can solve different types of nonlinear problems in image classification and retrieval process. After that majorly focus on research learning methodologies for improve performance of the Multi Slice CT Angiogram images.

So, in this research is summarizing the problem with liner of RBF neural network, Non-Liner SVM and RBF NN with liner and non-liner. Final, RBF NN with liner and non-liner provided more value and proved as best compare with other existing methodologies. This methodology consumes less time for both learning as well as testing comparatively than any other methods like back propagation. This issue drastically improves the estimation efficiency and accuracy for real time 128, 256 slices CT scan angiogram images.
\end{abstract}

\section{INTRODUCTION}

Nowadays medical systems are providing lot of images for diagnosis problem in right way and treatment process as well. It also links 'classical' diagnosis to modern forms of treatment such as image guided surgery. Major number of human beings is suffering with heart artery disease. Most of medications processes are not able to identify blocked areas of a blood vessel in heart soon [1] [2]. Earlier and nowadays most of the heart Patients undergoing a coronary CTA scan receive an iodinecontaining contrast material (dye) as an intravenous injection to ensure the best possible images and allow reproducible and accurate non-invasive evaluation of coronary artery diseases. CT scans of internal organs, bones, soft tissue and blood vessels provide greater clarity and reveal more details than regular x-ray exam. Basic CTA scan 3D image is shown below Fig.1 [3].

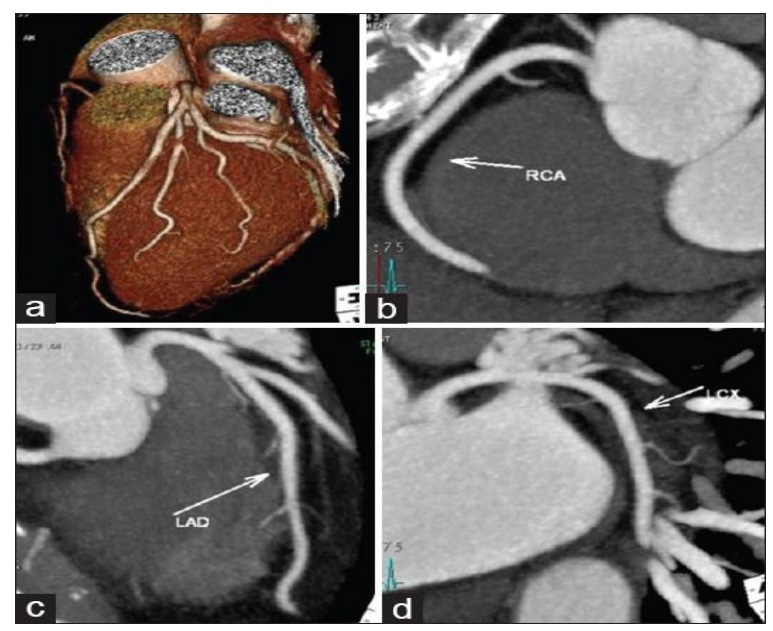

Figure 1: Basic heart CTA scan 3D image

Preoperative coronary computed tomography (CT) angiography is shown below figure 2 . Figure 2 shows the four images. Image A represents the location of the leak in the left ventricle is the sub-mitral valve prosthesis area. Image B represents the three large lobulated aneurysms are seen bulging (an excessive localized swelling of the wall of an artery) from the base of the left ventricle with calcified walls. Images $\mathrm{C}$ and $\mathrm{D}$ represents the Three-dimensional reconstruction of preoperative coronary CT angiography [4] [5]. PsA - Pseudo Aneurysm; LV - 
Left Ventricle; AV- Aortic Valve; MV - Mitral Valve; Dao - Descending Aorta. Based on this, we diagnosed the patient with a pseudo aneurysm of the left ventricle, and surgical treatment was planned [6] [7].
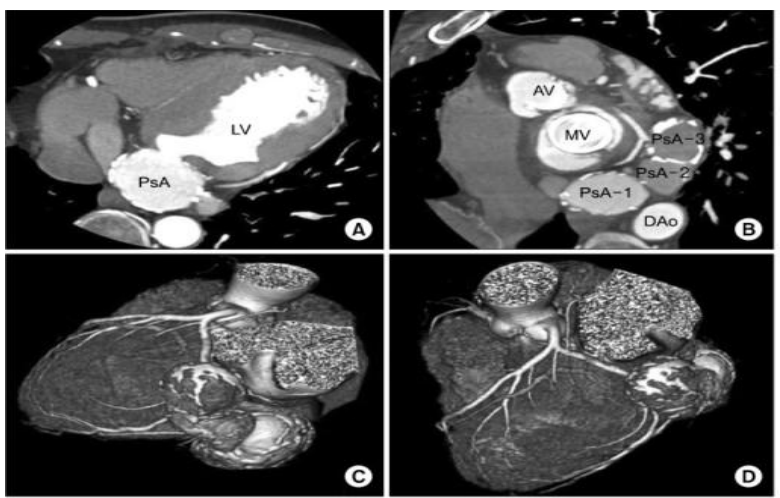

Figure 2: Basic multi slice CT scan angiogram image

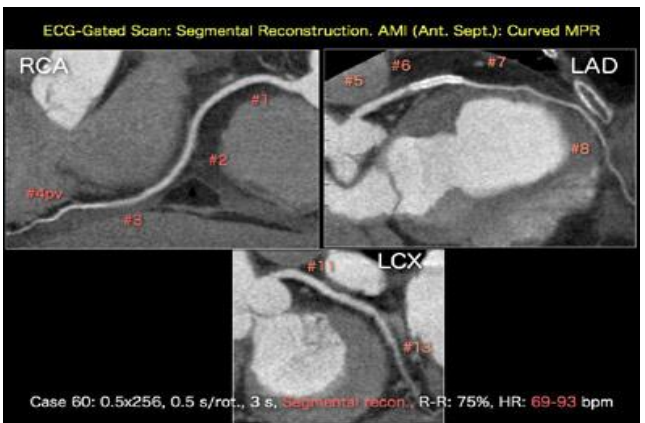

Figure 3: Basic segmental reconstruction of CT scan 3D image

In process of medical analysis CT images used normal first step as segmentation. Segmentation is going on agile fashion the research for medical image analysis. Now, CT scanner most advanced and using 128, 256 slices CT scanners are providing the most valuable information for processing. Several general-purpose algorithms and techniques have been developed for image segmentation. Multi slice CT angiogram segmentation is performed with the help of k-means++ algorithm. Segmental reconstructions of angiogram scan shows high-resolution images of (clockwise from top) the right coronary artery, left anterior descending artery, and left circumflex artery acquired on a multi slice CT scanner[8] [9].

Features are an important measurement for image understanding. A complete feature representation of the segmented region is an important issue for object classification and analysis. After the segmentation and feature extraction next step is to decide whether the patient angiogram waveform has disease or not. For that, Support Vector Machine method is used to confirm the presence of disease. Here SVM is non-liner method when use any type of application will miss most liner ways of blood vessels and lack of speed in process [10]. Basic SVM method is most supported for binary classification. Recently SVM implemented for multi class classification problems. As per details from the WIKI, SVMs are also useful in many applications to classify with up to $90 \%$ of the compounds classified correctly. Many recent methods are used to extended SVM from binary classification to multi class classification problem, in that main popular methods are four: One Against One (OAO), One Against All (OAA), Fuzzy Decision Function (FDF) and Decision Directed Acyclic Graph (DDAG).So, way of solution is with which method can solve the problem of linear and nonlinear classifiers. The best algorithm of liner and non-liner classifier is single layer Neural Network, i.e., Radial Based Function Neural Network. Final analysis of the research gives more improve of the accuracy in terms of the time and classifications images [11].

\section{BACK GROUND}

Now way days most of the humans suffer the hart problem. Multi slice CT scanner is used to identify heart disease. Multi-detector CT is convenient and reliable non-invasive imaging modality for assessment of human angiogram 3D images. Accurate heart segmentation is a decisive requirement for computer-aided hepatic disease diagnosis and treatment planning. If the segmentation can be performed rapidly, the results can also be used in intraoperative guidance. Manual annotation is error prone and time-consuming. Automatic hart segmentation from Computed Tomography (CT) volumes is therefore highly demanded. Computed tomography (CT) datasets facilitates evaluation of 3D CT angiogram images [12]. Multi-detector CT is considered convenient and reliable non-invasive imaging modality for assessment of human angiogram 3D images. This paper provides information on expanding your imaging services with multislice CT. First Multislice CT invention by Kalender in the 1980s; Kalender argues that the terms spiral and helical are synonymous and equally acceptable. Spiral computed tomography is a computed tomography (CT) technology in which the source and detector travel along a helical path relative to the object. Typical implementations involve moving the patient couch through the bore of the scanner whilst the gantry rotates. Spiral CT can achieve improved image resolution for a given radiation dose, compared to individual slice acquisition [13].

A scanner with more slices allows faster acquisition and makes it easier to examine uncooperative children and frailer older patients. If no of slices or increased the speed of the scan also increased. And it gives the perfect visual of the heart, since 20th century first introduce the 16 slice CT 
scanner, after that 64 slice CT scanner .The multi slice CT angiogram with 16, 32, 64 are facing problem in the issue of quantitative evaluation of segmentation of the san result. The research goes with segmentation which is one of the difficult tasks in image processing and determines the outcome of analysis and evaluation of pathological regions. The main objectives of this research are to improve the accuracy of the multi slice CT scan with 128,256 slices for advanced image processing [14].

SVM is a Soft Computing system developed by Dr. Vapnik (1995) in Bell Lab. Its basic concept is to construct the best super plane in sample space so that the margin between super plane and the sample set of different types will be a maximum.

\section{PROPOSED METHODOLOGY}

This research proposed methodology focuses on identification of different existing and new ways of extracting features of Multi Slice CT Images. The main aim is to provide a method for recognizing and classifying CT Angiogram images using liner, non-liner and both classifier. Proposed methodology is shown Fig. 4.

The main objectives of this work is

- To study and review of Multi Slice CT Angiogram Images

- Segmentation of Angiogram images with high rated level.

- Feature extraction with Colour, Texture and relational of analysis.

- Analysing the Machine Learning Algorithms with current research trends and developed the following algorithms to find the disease from Multi Slice CT Angiogram Images:

- First Step is to analysis use existing method Support Vector Machine (SVM)

- In second step, used Liner and Non-Liner RBF Neural Network for analysis problem.

- To Test on different data sets of multi-slice CT Angiogram Images and summarize the analysis of accuracy for the above proposed algorithms.

In this paper, exhibit best process for Multi Slice CT Angiogram image classification using liner, non-liner and both methodologies.

Major process divided into four steps:

- First step is the Data Processing is collecting data from different image datasets. This digital data is pre-processed before data selection.

- After data processing the entire data is grouped into different datasets. Each data set consists of Train Data sets and Test Data sets. Train data set is used to build the system and Test data set is used to measure the system.

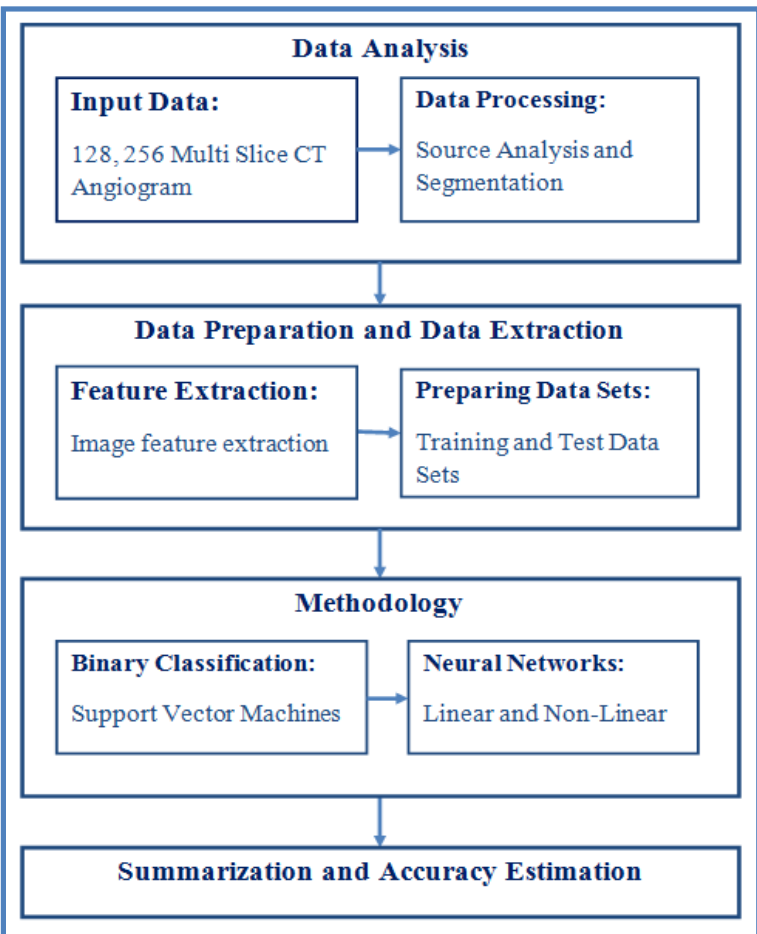

Figure 4: Block Architecture Proposed Methodology

- There are different methodologies proposed in this research. In the medical imaging analysis, features and measurements can also be used for region segmentation to extract meaningful structures, subsequently, interpret the result using classification methods.

- Following methods are used in current research areas: Support vector machines and neural networks.

- The final one is accuracy assessment, after finalizing the method build the system first and then test with different queries for evaluating the performance in terms of accuracy.

\subsection{Test Data}

In this Research, data is collected from one source, which is osirix-viewer. Osirix-viewer organization is help to do research for medical image open data. Data sets are provided in form of DICOM format and JPEG 2000. Our research source data is converted to binary and worked as with jpeg images.

Collected three types of data sets are from data source. About each data set, here explained below:

Agecanonix Dataset:122 records from 13 subjects this dataset contains, each voxel size is $0.47 \times 0.47 \mathrm{x}$ $0.50(\mathrm{~mm})$. Each signal is digitized at 1106 samples per second, with 16 bit resolution over a range of \pm $16.384 \mathrm{mV}$. Each subject is specified in below Table 1 with number of records. 
Sarvadeva BhatlaMurali Krishna. Int. Journal of Engineering Research and Application www.ijera.com ISSN: 2248-9622, Vol. 7, Issue 2, (Part-2) February 2017, pp.29-35

\begin{tabular}{|c|c|}
\hline Subject Name & No of Records \\
\hline CorCTA w-c 1.0 B20f -6 & 355 \\
\hline CorCTA w-c 3.0 B20f $0 \%-9$ & 51 \\
\hline CorCTA w-c 3.0 B20f $10 \%-10$ & 51 \\
\hline CorCTA w-c 3.0 B20f $20 \%-11$ & 51 \\
\hline CorCTA w-c 3.0 B20f $30 \%-12$ & 51 \\
\hline CorCTA w-c 3.0 B20f $40 \%-13$ & 51 \\
\hline CorCTA w-c 3.0 B20f $50 \%-14$ & 51 \\
\hline CorCTA w-c 3.0 B20f $60 \%-15$ & 51 \\
\hline CorCTA w-c 3.0 B20f $70 \%-16$ & 51 \\
\hline CorCTA w-c 3.0 B20f $80 \%-17$ & 51 \\
\hline CorCTA w-c 3.0 B20f DIASTOLE -8 & 119 \\
\hline CorCTA w-c 3.0 B20f SYSTOLE - 7 & 119 \\
\hline CorCTA w-o 3.0 B20f -4 & 58 \\
\hline
\end{tabular}

Table 1: Tested data from Agecanonix and \#records

FIVIX Dataset:FIVIX dataset 1754 records from 3 subjects. Below Table 2 subjects are specified with number of records.

\begin{tabular}{|l|r|}
\hline Subject Name & No of Records \\
\hline CorCTA 0.6 B20f $65 \%$ dias- 11 & 414 \\
\hline CorCTA 3.0 B20f $0-90 \% 10 \mathrm{ph}-13$ & 530 \\
\hline pet cardiac 10 gates Volume (Corrected) - 404 & \\
\hline
\end{tabular}

Table 2: Tested data from FIVIX and \#records

All subjects are 1754 simultaneously measure Multi slice CT angiogram heart images. Each voxel size is $0.35 \times 0.40 \times 0.40(\mathrm{~mm})$.

MAGIX dataset:MAGIX contains 760 simultaneously measure Multi slice CT angiogram heart images. Each voxel size is $0.40 \mathrm{x} 0.40 \mathrm{x}$ $2.0(\mathrm{~mm})$. All from 10 different subjects specified in below Table 3 .

\begin{tabular}{|c|c|}
\hline Subject Name & $\begin{array}{l}\text { \# of } \\
\text { Records }\end{array}$ \\
\hline $\begin{array}{l}\text { Cir CardiacCirc 3.0 B20f 0-90\% } \\
\text { RETARD_DECLECHEMENT 0 \% - } 10\end{array}$ & 76 \\
\hline $\begin{array}{l}\text { Cir CardiacCirc } 3.0 \text { B20f } 0-90 \% \\
\text { RETARD_DECLECHEMENT } 10 \%-10\end{array}$ & 76 \\
\hline $\begin{array}{l}\text { Cir CardiacCirc } 3.0 \text { B20f } 0-90 \% \\
\text { RETARD_DECLECHEMENT } 20 \%-10\end{array}$ & 76 \\
\hline $\begin{array}{l}\text { Cir CardiacCirc } 3.0 \text { B20f } 0-90 \% \\
\text { RETARD_DECLECHEMENT } 30 \%-10\end{array}$ & 76 \\
\hline $\begin{array}{l}\text { Cir CardiacCirc } 3.0 \text { B20f } 0-90 \% \\
\text { RETARD_DECLECHEMENT } 40 \%-10\end{array}$ & 76 \\
\hline $\begin{array}{l}\text { Cir CardiacCirc } 3.0 \text { B20f } 0-90 \% \\
\text { RETARD_DECLECHEMENT 50\% - } 10\end{array}$ & 76 \\
\hline $\begin{array}{l}\text { Cir CardiacCirc } 3.0 \text { B20f } 0-90 \% \\
\text { RETARD_DECLECHEMENT } 60 \%-10\end{array}$ & 76 \\
\hline $\begin{array}{l}\text { Cir CardiacCirc } 3.0 \text { B20f } 0-90 \% \\
\text { RETARD_DECLECHEMENT } 70 \%-10\end{array}$ & 76 \\
\hline $\begin{array}{l}\text { Cir CardiacCirc } 3.0 \text { B20f } 0-90 \% \\
\text { RETARD_DECLECHEMENT } 80 \%-10\end{array}$ & 76 \\
\hline $\begin{array}{l}\text { Cir CardiacCirc } 3.0 \text { B20f } 0-90 \% \\
\text { RETARD_DECLECHEMENT } 90 \%-10\end{array}$ & 76 \\
\hline
\end{tabular}

Table 3: Tested data from MAGIX and \#records

Here total 3 types of data set with 1106, 1754 and 760 Multi Slice CT images are used in this research. Next step of research is extract feature set from collection data set.

\begin{tabular}{|l|l|}
\hline Technique Name & Feature Name \\
\hline Statistical Pixel-Level & Mean \\
\hline Statistical Pixel-Level & Variance \\
\hline Statistical Pixel-Level & Gray Value \\
\hline Statistical Pixel-Level & Contrast of Pixel \\
\hline Statistical Pixel-Level & Edge Gradient \\
\hline Shape & Circularity \\
\hline Shape & Compactness \\
\hline Shape & Moments \\
\hline Shape & Chain Codes \\
\hline Relational & Relational Structure \\
\hline Relational & Hierarchical Structure \\
\hline
\end{tabular}

Table 4: Extract Features Descriptions

\subsection{Non-Liner: Support Vector Machine- RBF}

The original SVM algorithm was invented by Vladimir N. Vapnik in 1995. Support vector machines are supervised learning models with associated learning algorithms . A support vector machine constructs a set of hyper planes in an infinite-dimensional space, which can be used for classification, regression, or other tasks. In the case of support vector machines, a data point is viewed as a display style dimensional vector, and we want to know whether we can separate such points with a $\{(\mathrm{p}-1)\}$ dimensional hyper plane. This is called a linear classifier [15] [16].

Basic SVM hyper plane image is shown below Figure 5. Figure 5 shows the three hyper planes. 
1. H1 hyper plane does not support the classes. 2 . Hyper plane $\mathrm{H} 2$ does, but only small margin. 3. Hyper plane H3 separates them with the maximum margin.

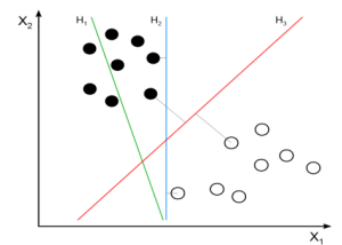

Figure 5: SVM Hyper Plane

In a finite dimensional space set of hyper planes are not linearly separable, so it is called as Non-linear classifier. Non-linear classifier working is based on kernel method.

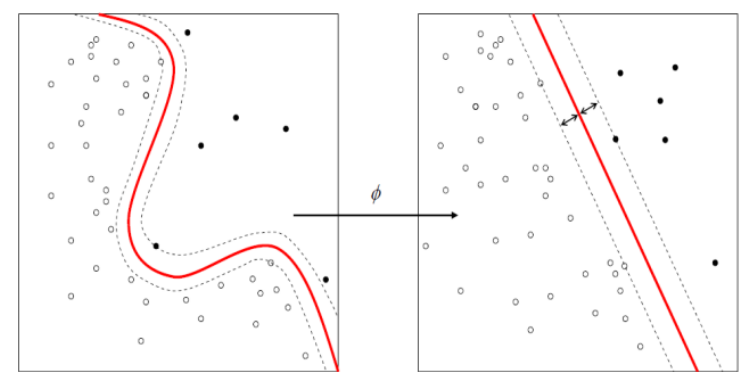

Figure 6: Traditional Radial Basis Function Network outline

Mainly in non-liner SVM using different kernel methods to train data sets. Following kernel methods using major popular:

- Homogeneous -Polynomial

- Inhomogeneous - Polynomial

- RBF - Gaussian Radial Basis Function

- HT - Hyperbolic Tangent

A common disadvantage of non-parametric techniques such as SVMs is the lack of transparency of results. SVMs cannot represent the score of all companies as a simple parametric function of the financial ratios, since its dimension may be very high. It is neither a linear combination of single financial ratios nor has it another simple functional form. The weights of the financial ratios are not constant. Thus the marginal contribution of each financial ratio to the score is variable. Using a Gaussian kernel each company has its own weights according to the difference between the value of their

\section{ACCURACY ASSESSMENTS}

In this paper of research is used one source with three different data sets. About data sets are specified in section 3.1. Total data sets are three types, done research with final output is find the place of blood vessels in images. Each data set, own financial ratios and those of the support vectors of the training data sample [17].

\subsection{Liner and Non-Liner: RBF Neural Network}

Figure 2.4, shows the block diagram of RBF Neural network. $\mathrm{x} 1, \mathrm{x} 2, \ldots \mathrm{Xd}$ are input vector and wi are the weights among hidden and output layer. The output function $\mathrm{f}$ compute by using the below approximation function like below equation (1).

$y(x)=\sum_{i=1}^{n}$ wi $\varphi(|| x-x i||)-(1)$

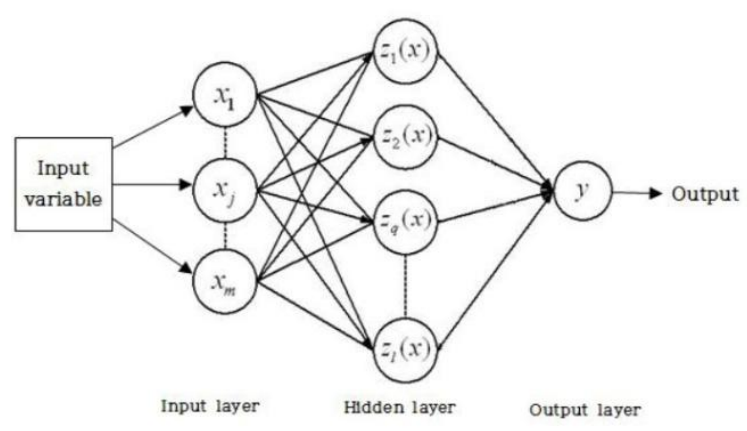

Figure 7: Block diagram of RBF neural network

Different optimization methods are used for find weight values in Radial Basis Function Neural Network. Most popular method is Gradient Descent. GD is an optimization algorithm used for calculating a local minimum of a function. The algorithm takes steps proportional to the negative of the gradient of the function at the current point. RBF output written as below equation (2):

$\mathrm{y}=\mathrm{w} \cdot \mathrm{h} \quad--(2)$

Where $\mathrm{w}$ is defined as weight matrix, $\mathrm{h}$ is hidden layer output values. Here supervised leaning we already know output values y based on below function way, used to find the w value with below equation (3):

$$
\mathrm{w}=\mathrm{h} \cdot \mathrm{y}^{\wedge}(-1)
$$

Here in this paper of research, used weight value used matrix inversion showed above equation (3). Above two methods are discussed following next section, and its proved RBF NN accuracy is more compare than SVM -RBF [18].

defined some set of data has training data set and left our part is test data set. Instance of data set is already defined how much blood vessels part of data sets for train data. Has first training data set is defined $90 \%$ data set from original data and $10 \%$ consider has test data set. Like that, decrease training data set $\%$ and increase test data set $\%$ with different 7 cases are 
divided as data sets and showed detailed each case in below Table 5 .

\begin{tabular}{|l|c|c|}
\hline $\begin{array}{l}\text { Seq. No } \\
\text { Case }\end{array}$ & $\begin{array}{l}\text { Training Data Set } \\
\text { \% from Original } \\
\text { Data Set }\end{array}$ & $\begin{array}{l}\text { Test Data Set \% } \\
\text { from Original } \\
\text { Data Set }\end{array}$ \\
\hline Case \#1 & 90 & 10 \\
\hline Case \#2 & 80 & 20 \\
\hline Case \#3 & 70 & 30 \\
\hline Case \#4 & 60 & 40 \\
\hline Case \#5 & 50 & 50 \\
\hline Case \#6 & 40 & 60 \\
\hline Case \#7 & 30 & 70 \\
\hline
\end{tabular}

Table 5: Training and Test Data Sets \% with Different Test Cases

Used with above training and test data sets for each analysis division of \% shown in above Table 5. Assessment of accuracy done for following methods with above train and test data set cases:

- Support Vector Machines With RBF

- Neural Network with RBF

\begin{tabular}{|l|c|c|}
\hline Cases & $\begin{array}{l}\text { Accuracy of } \\
\text { SVM-RBF }\end{array}$ & Accuracy of RBF NN \\
\hline Case \#1 & $94 \%$ & $96 \%$ \\
\hline Case \#2 & $91 \%$ & $95 \%$ \\
\hline Case \#3 & $85 \%$ & $95 \%$ \\
\hline Case \#4 & $87 \%$ & $95 \%$ \\
\hline Case \#5 & $82 \%$ & $90 \%$ \\
\hline Case \#6 & $75 \%$ & $86 \%$ \\
\hline Case \#7 & $70 \%$ & $85 \%$ \\
\hline
\end{tabular}

Table 6: Accuracy Assessment for proposed Methodologies

Accuracy is exhibited above Table 6 for research methodologies. Clear proved $\mathrm{RBF}-\mathrm{NN}$ is more accurate compare than SVM-RBF and another thing is here clear specified even decrease train data set also accuracy is stabilized in all aspect not much decrease as like SVM-RBF [18].

Additional analyzed SVM RBF stabilized different with Sigma and Cost values. Final best Sigma and Cost value: 1.16 and 0.05 . Additional analyzed RBF NN stabilized different with Weights and Bias values. Total weight values are 132 values and 2 bias values.

\section{CONCLUSIONS}

This paper, proposed a method to find the Heart diseases. In this research using Computed Tomography (CT) scanner for identified blood vessel in heart area. The problem of identification blood vessels in heart is like most binary classification. Majorly binary classification algorithms are classified in two forms, linear and nonlinear. Max of the times non-liner classification algorithms are best comparing with linear algorithms for large data sets like Images, Application Big Data. And most popular in binary and non-liner classification algorithm is Support Vector Machine (SVM), and it is most successfully industry algorithm. Here SVM is non-liner method when use any type of application will miss most liner ways of blood vessels and lack of speed in process. So, way of solution is with which method can solve the problem of linear and nonlinear classifiers. The best algorithm of liner and non-liner classifier is single layer Neural Network, i.e., Radial Based Function Neural Network.

Future extended with advanced methodology for image classification, feature extractions and best accuracy assessment.

\section{REFERENCES}

[1]. Lee H, Lee KS, Sim SB, Jeong HS, Ahn HM, Chee HK, Korean J Thorac Cardiovascular Surg. "Left ventricular pseudo aneurysm after valve replacement" Published in 2015 Feb;

[2]. Wang M, Qi HT, Wang XM, Eur J Radiol. "Dual source CT versus single source CT in cardiac CT angiography" published in 2009; 72:396-400.

[3]. Baumüller S,Leschka S, Desbiolles L. "Angiography at lower heart rates comparison of accuracy and radiation". Radiology 2009; 253:56-64.

[4]. Guo SL, Guo YM, Zhai YN. "Diagnostic accuracy of first generation dual-source computed tomography in the assessment of Image Segmentation and Feature Extraction ofcoronary artery disease".Int J Cardiovascular Imaging 2011; 27:755-71.

[5]. Lei Z, Han P, Xu H, Yu J. “Application study of 512-slice computed tomography low dose coronary angiography”. Digit Med 2015; 1:28-33.

[6]. A. P. Dhawan. "Coronary artery diseaseCT Angiography". World Scientific Publishing Co. Pte. Ltd, 2008, pp 197-228.

[7]. Andrej Krenker, J Bester, and Andrej Kos. "Introduction to the artificial neural networks". ISBN, pages 978-953, 2011.

[8]. J. Kennedy, R.C. Eberhart, Y. Shi.“Swarm Intelligence”.Morgan Kaufmann Publishers in 2001 .

[9]. JinGu, He-shui Shi, Ping Han, Jie Yu, Guina Ma \& Sheng Wu. "Image Quality and Radiation Dose for Prospectively Triggered Coronary CT Angiography". Scientific 
reports 6, article number: 34795(18 October 2016).

[10]. Dr. Kazuhiro Katada, chairman of the department of radiology at Fujita Health University School of Medicine in Japan.

"256-slice CT at Stanford University's International Symposium on Multi detector

Row CT technology”.Autominnie.com during the sanfranciscomeeting. August 21 2006.

[11]. NarendraKohli, Nishchal, K.Verma "Arrhythmia classification using SVM with selected features".International Journal of Engineering, Science and TechnologyVol. 3, No. 8, 2011, PP 122-131.

[12]. Nico R Mollet, Filippo Cademartiri, Pim J de Feyter Heart."Non-invasive multislice CT coronary imaging”. 2005 Mar; 91(3): 401407. Doi: 10.1136/hrt.2004.034009 PMCID: PMC1768758

[13]. Knez A, Becker CR, Leber A, et al. Usefulness of multislice spiral computed tomography angiography for determination of coronary artery stenoses. Am J Cardiol 2001; 88:1191-4. [PubMed]

[14]. Vogl TJ, Aboimaali ND, T Diebold.“Multidetector row CT coronary angiography”. Radiology 2002; 223:212-2, PubMed.

[15]. A. P. Dhawan and S. Dai. "Clustering and Pattern Classification”, in Principles and Advanced Methods in Medical Imaging and Image Analysis. Singapore". World Scientific Publishing Co. Pte. Ltd, 2008, pp 229-265.

[16]. Flohr TG, Schoepf UJ, Kuettner A. Advances in cardiac imaging with 16section CT systems. AcadRadiol 2003; 10:386-401, PubMed.

[17]. Budoff, M.J., Achenbach, S., Blumenthal, R.S., Carr, J.J., Goldin, J.G., Greenland, P., Guerci, A.D., Lima, J.A., Rader, D.J., Rubin, G.D., Shaw, L.J., Wiegers, S.E. "Assessment of coronary artery disease by cardiac computed tomography".
Circulation 114 , (2006)CrossRef

$1761-1791$

[18]. Syeda-Mahmood, T., Wang, F., Beymer, D., Amir, A., Richmond, M., Hashmi, S. Aalim."Multimodal mining for cardiac decision support”. Comput. Cardiol. 34, 209-212 (2007). 